\title{
UNSUPERVISED MORPHOLOGICAL SEGMENTATION FOR IMAGES
}

\author{
Philippe Salembier \\ Department of Signal Theory and Communications \\ E.T.S.E.T.B - Universitat Politecnica de Catalunya \\ Apdo. 30502, 08080 Barcelona, Spain
}

\begin{abstract}
This paper deals with a morphological approach to unsupervised image segmentation. The proposed technique relies on a multiscale Top_Down approach allowing a hierarchical processing of the data ranging from the most global scale to the most detailed one. At each scale, the algorithm consists of four steps: image simplification, feature extraction, contour localization and quality estimation. The main emphasis of this paper is to discuss the selection of a simplification filter for segmentation. Morphological filters based on reconstruction proved to be very efficient for this purpose. The resulting unsupervised algorithm is very robust and can deal with very different type of images.
\end{abstract}

\section{INTRODUCTION}

Segmentation is the process that partitions images into homogeneous and connected regions [1]. This definition is not very precise and the homogeneous term allows different interpretations of its meaning. In the past, a large number of algorithms have been proposed. Most of them rely on three steps: preprocessing, feature extraction and decision. The preprocessing simplifies the original signal which is too complex to be processed at once. The feature extraction intends to extract the pertinent parameters assessing the homogeneity of the regions. Finally, the decision defines precisely the contours of the regions.

This study investigates a unsupervised Top-Down algorithm relying on morphological techniques. It is an extension of the work reported in [2] where it has been shown that mathematical morphology offers very efficient and powerful simplification, feature extraction and decision tools. The main emphasis of this paper is to precisely investigate the quality of morphological filters for simplification in the context of segmentation. The organization is as follows: the next section discusses the general multilevel structure of the algorithm. Section 3 is devoted to the presentation of the morphological filters of interest. The algorithm involves four basic steps: preprocessing, feature extraction, decision and quality estimation which are described in section 4. Finally, section 5 is devoted to results and discussions.

\section{HIERARCHICAL STRUCTURE}

The segmentation algorithm proposed in [2] follows a Top-Down procedure. The algorithm starts by considering the whole image as a single region. The first level computes a simplified segmentation, then the successive levels improve the current segmentation by introducing new regions.

All hierarchical levels are based on the structure described in Fig. 1, involving preprocessing, feature extraction and decision steps. Let us recall that the objective of the preprocessing is to simplify the data to make them easier to segment. In section 5, the simplification criterion will be discussed in detail. After simplification, a feature extraction assesses the homogeneity. In morphology, the feature extraction is known as marker extraction [3]. A marker is a binary signal indicating the presence of an homogeneous area. It does not try to precisely locate the contours. This is the goal of the decision step.

The current segmentation will be improved by the next hierarchical level which should deal with the components that have not been properly segmented. This remark leads to the use of a fourth step aimed at the quality estimation of the current segmentation. The quality estimation should indicate the areas which have not been properly segmented. This can be achieved by actually modeling each region, that is by filling each region with a grey level model, and by computing the difference between the modeled and the original images. Let us call this difference image the modeling residue.

The whole hierarchy is a succession of blocks as the one described in Fig. 1. The information which is transmitted between two levels is the residue, the segmentation result and the original image. On the first level, the modeling residue is defined as the original image itself and the segmentation result is composed of a single region. The multilevel approach of the algorithm relies on the simplification step which controls the amount of information which is kept for segmentation. On the first level, this simplification discards the major part of the information. Then, the simplification is progressively reduced to get a more precise result. As it constitutes the heart of the hierarchy, the main part of this study is dedicated to the selection of a simplification filter.

\section{MORPHOLOGICAL FILTERS}

This section briefly describes morphological tools used for the algorithm. Only the notions of interest here will be presented and more details about mathematical morphology can be found in [4]. 


\subsection{Basic notions}

Mathematical morphology relies on a structure called complete lattice. Basically, a complete lattice is a set of elements on which an ordering relation ( $\leq$ ) and two laws, supremum and infimum, are defined. The simplest lattice used to deal with discrete grey level signals is the lattice of grey level functions. The order is defined at each spatial position by the natural ordering of grey level values:

Two functions $f, g$ are such that $f \leq g$ if for each spatial position $x, f(x) \leq g(x)$

For discrete signals, the infimum is the minimum and the supremum the maximum. The first transformations to be defined on this lattice are those preserving the structure, that is the ordering relation $\leq$, and commuting with one of the two laws. This approach leads to the definition of increasing transforms, erosions and dilations:

Preserve of the structure: $\quad f \leq g \Rightarrow \psi(f) \leq \psi(g)$

These transformations are called increasing

Commute with Max: $\quad \psi(\operatorname{Max}\{\mathrm{f}, \mathrm{g}\})=\operatorname{Max}\{\psi(\mathrm{f}), \psi(\mathrm{g})\}$

These transformations are called dilations

Commute with Min: $\quad \psi(\operatorname{Min}\{f, g\})=\operatorname{Min}\{\psi(f), \psi(g)\}$

These transformations are called erosions

For the sake of simplicity, we are going to present only two examples of dilation (and erosion) that will be useful for the algorithm:

Dilation and erosion with flat structuring element

If $f\left(x_{i}\right)$ denotes an $N$ dimensional signal and $M_{n}$ a window or flat structuring element of size $n$, the erosion and dilation are respectively given by:

$\varepsilon_{n}(f)\left(x_{i}\right)=\operatorname{Min}\left\{f\left(x_{i+k}\right), k \in M_{n}\right\}$

$\delta_{n}(f)\left(x_{i}\right)=\operatorname{Max}\left(f\left(x_{i-k}\right), k \in M_{n}\right\}$

More general definitions of erosions and dilations with non flat structuring element can be found in the literature [4]. Here, flat structuring elements are preferred because they preserve edges, allow fast implementation and are robust with respect to grey level changes (in particular, they commute with anamorphosis). Erosion and dilation are dual in the sense that:

$\delta_{n}(f)=-\varepsilon_{n}(-f)$

\section{Geodesic grev level dilation and erosion:}

A geodesic transform [5] of a function ( $f$ ) is defined with respect to a reference function ( $r$ ). The geodesic dilation of size one (smallest size on the digital space) is defined as the minimum between the dilation of size one of the function ( $f$ ) and the reference ( $r$ ). The geodesic erosion is defined by duality:

$\delta(1)(f, r)=\operatorname{Min}\left\{\delta_{1}(f), r\right\}$

$\varepsilon^{(1)}(f, r)=-\delta(1)(-f,-r)$

The justification of this terminology involving the notion of geodesy goes beyond the scope of this description and the reader is referred to [5]. Geodesic dilations and erosions of large size are defined by iterations. For example, the geodesic dilation (resp. erosion) of infinite size, also called reconstruction by dilation (resp. by erosion) can be expressed by:

$\gamma^{(\mathrm{rec})}(\mathrm{f}, \mathrm{r})=\delta^{(\infty)}(\mathrm{f}, \mathrm{r})=\ldots \delta^{(1)}\left(\ldots \delta^{(1)}(\mathrm{f}, \mathrm{r}) \ldots, \mathrm{r}\right)$

$\varphi^{(\mathrm{rec})}(\mathrm{f}, \mathrm{r})=\varepsilon^{(\infty)}(\mathrm{f}, \mathrm{r})=\ldots \varepsilon^{(1)}\left(\ldots \varepsilon^{(1)}(\mathrm{f}, \mathrm{r}) \ldots, \mathrm{r}\right)$

Note that the implementation of these functions can be done very efficiently avoiding any iterating process and leading to extremely fast algorithms [6].

\subsection{Morphological filters}

A morphological filter is any increasing and idempotent transformation. If the transformation is in addition antiextensive (resp. extensive), it is an algebraic opening (resp. algebraic closing). Let us recall the definition of these properties:

$\psi$ is idempotent if for all $\mathrm{f}, \psi(\psi(\mathrm{f}))=\psi(\mathrm{f})$

$\psi$ is extensive if for all $f, f \leq \psi(f)$

$\psi$ is antiextensive if for all $f, \psi(f) \leq f$

\section{Morphological opening and closing:}

The most classical cases of opening and closing are based on composition of erosion and dilation with structuring element. They are called morphological:

Morphological opening: $\gamma_{n}=\delta_{n} \varepsilon_{n}$,

Morphological closing: $\varphi_{n}=\varepsilon_{n} \delta_{n}$

The opening (resp. closing) simplifies by removing the bright (resp. dark) components which do not fit within the structuring element. If the simplification has to deal with both bright and dark elements an open_close $\left(\gamma_{n} \varphi_{n}\right)$ or a close_open $\left(\varphi_{n} \gamma_{n}\right)$ can be used. None of these filters are self-dual, but in practice they approximately remove the same kind of information. They can be used to simplify the image before feature extraction, but they do not allow a perfect preservation of the contour information. In order to improve the contour preservation properties, two different approaches have been investigated in the past. The first one consists in combining several morphological openings or closings, and the second one involves reconstruction processes.

\section{Directional opening and closing:}

Suppose that several morphological openings are computed in parallel and that, for each pixel, the final result is equal to the maximum of the various openings. This new transformation is an algebraic opening. Finally, if the various openings are computed with line segments of a given size but all possible orientations, the opening is called directional. By duality, a directional closing can be defined. These filters simplify by removing the components that do not fit in one of the line segments. 
As a result, they preserve elongated objects and corrupt less contours than morphological opening or closing.

\section{Opening and closing by reconstruction:}

Morphological filters involving reconstruction process are attractive for segmentation because they lead to very good contour preservation [7]. The opening by reconstruction of opening (a closing is obtained by duality) is defined by:

Opening by rec. of opening: $\gamma^{(\mathrm{rec})}\left(\gamma_{n}(f), f\right)$

Closing by rec. of closing: $\varphi^{(\mathrm{rec})}\left(\varphi_{\mathrm{n}}(\mathrm{f}), \mathrm{f}\right)$

The simplification is performed by the opening (closing) which eliminates all components smaller than the structuring element, and the reconstruction restores the contour of the components which have not been totally removed by the opening (closing).

\section{BASIC SEGMENTATION STEPS}

\subsection{Image simplification}

As the simplification filter controls the amount of information which is removed before feature extraction and decision, it defines the notion of hierarchy in the segmentation. This section is devoted to the study of this filter. In a first step, a criterion is defined. Then, the performances of morphological filters are discussed.

\section{Simplification criterion:}

Assume that a grey level segmentation algorithm is used after simplification (this restriction is done to avoid complex and time consuming texture segmentation). For such an algorithm, an easy image to segment is composed of regions of constant grey level value and sharp contours corresponding precisely to those of the original image. To assess the quality of a filter, one can use a synthetic image composed of several objects on a background and corrupted by noise. The advantage of using a synthetic image is that the optimal segmentation result is a priori known. This image is simplified by the filter and then segmented using the watershed transform [3]. Two parameters are measured after segmentation:

- The number of pixels differing between the actual and the optimal segmentation: this parameter, called Edge Localization, measures the property of contour preservation of the filter.

- The variance of the simplified signal inside each segmented region: this feature, called Flatness, assesses the filter efficiency to produce flat, and therefore easily segmentable, regions.

Each measure is plotted on the Edge localization/Flatness plane. For each filter, a set of measures are obtained by modifying the window (or structuring element) size. This creates a curve in the plane. Note that this approach is similar to the one reported in [8]. The main difference relies in the Flatness criterion used here because the filter is studied in the context of segmentation. Finally, tests have been performed with various noise pdf: Laplacian, Gaussian and IID. As they lead to similar conclusions, the results with Gaussian noise are presented here.

\section{Simplification performance.}

Fig. 2 presents results comparing the performances of a linear Gaussian filter with three morphological filters. Ideally, a good simplification filter should have low Edge localization as well as Flatness parameters. Its curve should lie close to the origin.

The worst filter is the linear filter. It does not preserve contours nor produces flat regions. This result, in accordance with intuition, justifies the use of nonlinear filters. The morphological open_close filter achieves a much better flatness but does not perfectly preserve contours. As discussed above, directional open_close filters try to improve the contour preservation. Fig. 2 demonstrates that it actually achieves a much better edge localization than the morphological open_close. Finally, the best filter for both criterion is the open_close by reconstruction which gives flat regions with perfectly localized contours. This test has also been performed with the median filter. Its performances are similar to that of the morphological open_close but at a much higher computational load (an open_close filter can be implemented in such a way that the computational load is independent of the size of the structuring element).

Fig. 3 presents the same kind of results but with Alternating Sequential filters: If $\mathrm{M}_{\mathrm{n}}$ denotes an open_close filter involving a structuring element of size $\mathrm{n}$, its alternating sequential version is defined as:

$M_{n}\left(M_{n-1}\left(\ldots M_{k} \ldots\left(M_{1}().\right)\right)\right)$

As far as the relative performances are concerned, the results of Fig. 3 are similar to the previous ones. In the average, alternating sequential filters are better. For morphological and directional open_close filters, the major improvement concerns the flatness of the region. The open_close by reconstruction still exhibits the best performances for both parameters. The major advantage of using the alternating sequential approach is the robustness with respect to the structuring element size.

Fig. 4 compares an open_close by reconstruction, its alternating sequential version and a simplified scheme where only two sizes involved in eq. (9) are computed: $M_{n}\left(M_{n / 2}().\right)$

The results are close to that of alternating sequential filters but at a severely reduced computational load.

These experiments allow the selection of simplification filters for segmentation. For a segmentation algorithm, a good complexity / performances filter is based on open_close by reconstruction with a simplified alternating sequential version. 
In the following, the feature extraction, decision and quality estimation steps are briefly described, more details can be found in [2].

\subsection{Feature extraction: Marker selection}

The feature extraction produces markers that are binary signals identifying the presence of homogeneous regions. This step takes into account the characteristics of the preceding simplification. As shown previously, the open_close by reconstruction produces constant grey level regions with abrupt contours. This remark leads to a double feature extraction: one extracting constant grey level regions and another one looking for high contrast.

Marker for constant regions: The areas where the morphological gradient has a low grey level value identify flat regions. This gradient is defined as:

$g=\delta_{1}(f)-\varepsilon_{1}(f)$

All connected regions of the gradient image having a grey level value lower than a threshold are considered as markers. This approach gives interesting results, however it should be completed by another technique. Indeed, Fig. 5. shows an original signal made of three regions and two transitions. One transition is very abrupt and detected by the gradient thresholding. However, the second transition, rather smooth, is not detected. To solve this problem, a feature extraction based on the grey level value and not the gradient may be used. This is the goal of the following technique.

Marker for contrasted regions: The marker extraction based on contrast criterion can be achieved with an extension of the "Top Hat" transform. In its self-dual version, this transformation can be viewed as the absolute difference between the original signal and the morphological center of: Id, $\gamma_{n} \varphi_{n}$, and $\varphi_{n} \gamma_{n}$ :

IId $-\operatorname{Min}\left(\gamma_{n} \varphi_{n}, \operatorname{Max}\left(\varphi_{n} \gamma_{n}\right.\right.$, Id $\left.)\right)$ ।

This transform is illustrated in Fig. 6, where it can be seen that the open_close $\left(\varphi_{n} \gamma_{n}\right)$ and close_open $\left(\gamma_{n} \varphi_{n}\right)$ filters generate upper and lower noise envelopes allowing a very reliable feature extraction.

Once extracted, the various marker images are merged together with the previous segmentation result by taking the union of all binary signals. A cleaning step involving a closing with reconstruction of small size is necessary to remove small artefacts (see [2]).

\subsection{Decision with the watershed algorithm}

Once the markers have been defined, the decision can be done by the watershed algorithm. The reader is referred to $[3,6]$ for more details about this algorithm. Let us briefly mention that the watershed defines the crest (bright) lines of an image. To get the contours of the object, it should work on the morphological gradient of the original signal. In fact, the direct segmentation of the gradient by the watershed results in an extreme oversegmentation. However, the gradient can be modified to segment only the objects which were "marked" by the feature extraction.

\subsection{Quality estimation by modeling}

The quality estimation computes the modeling residue created by filling each region with a grey level model and by computing the difference between the original and modeled images. The model choice has a rather strong influence on the remaining levels. From our experience, a model able to represent complex grey level functions leads to final segmentation with fewer regions and to a more accurate representation of the image. In section 5 , a first order polynomial model will be used as an example.

\section{RESULTS AND CONCLUSIONS}

The first example is shown in Fig. 7. It represents the successive contour (7.a), modeled (7.b) and residue images (7.c) of a camera man. The whole decomposition is obtained in four levels. At each level, the segmentation is improved by introducing new regions whereas the contours of the existing ones are perfectly preserved. In the mean time, information is extracted from the segmentation residues which ideally should tend to zero. At each level, the magnitude of simplification is reduced: for the example of Fig. 5, the following sizes of structuring elements for the simplification filter have been used: $60 * 60,30 * 30,10 * 10,3 * 3$.

Finally, Fig. 8 shows two other examples of segmentation. Only, the last segmentation level is presented.

As demonstrated by those examples, this unsupervised segmentation technique is robust and can deal with very different types of images. It produces several segmentation results from the simplest to the most complex one. This feature may be of interest in applications such as hierarchical recognition or progressive segmentation-based coding. Morphological tools have proved to be very efficient for simplifying and segmenting. A special attention has been paid to the quantitative evaluation of simplification filters in the context of segmentation. In this framework, morphological filters based on reconstruction processes are very attractive because they simplify the image while preserving the contour information and producing constant grey level regions.

\section{ACKNOWLEDGMENT}

The author is very grateful to Prof. J. Serra and A. Bangham for their discussions and comments. 


\section{REFERENCES}

[1] T. Pavlidis, Structural pattern recognition, SpringerVerlag, 1977.

[2] P. Salembier and J. Serra, Morphological multiscale segmentation of images, in Proc. of SPIE VCIP'92, Boston, MA, pp., 1992.

[3] F. Meyer and S. Beucher, Morphological segmentation, in Journal of VCIR, Vol. 1, No.1, September, pp. 21-46, 1990.

[4] J. Serra, Image analysis and mathematical morphology, Academic Press, Vol. I\&II, 1982-1988.

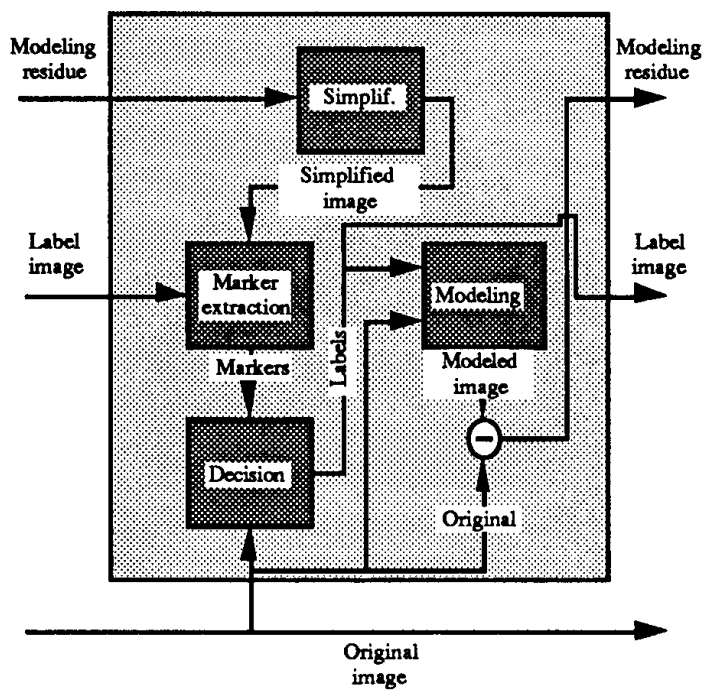

Figure 1: Hierarchical structure for segmentation

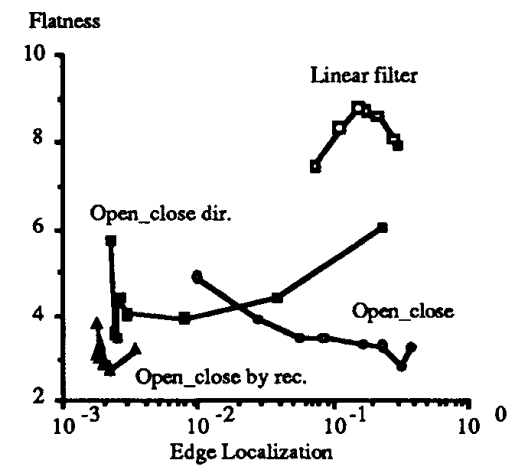

Figure 3: Edge versus Flatness performances of alternating sequential filters
[5] C. Lantuejoul and F. Maisonneuve, Geodesics methods in image analysis, in Pattern Recognition. Vol. 17, pp. 117-187, 1984.

[6] L. Vincent, Algorithmes morphologiques a base de files d'attente et de lacets, Extension aux graphes, PhD Thesis, School of Mines, 1990.

[7] P. Salembier and $M$. Kunt, Size-sensitive multiresolution decomposition of images with rank order based filters, in Signal Processing, Vol 27, No 2, pp. 205-241, May 1992.

[8] P. Salembier, J. Serra and A. Bangham, Edge versus contrast estimation of morphological filters, in Proc. of ICASSP'93, Mineapolis, MI, 1993.

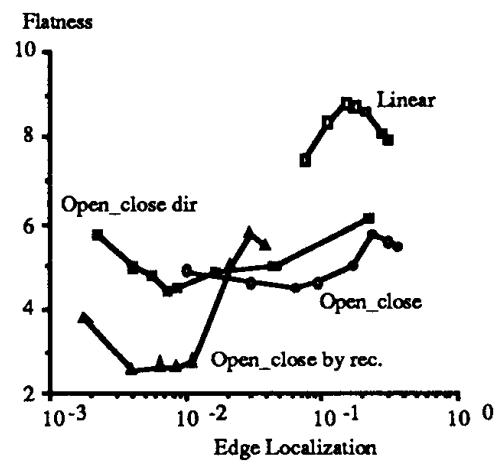

Figure 2: Edge versus Flatness performances of simplification filters

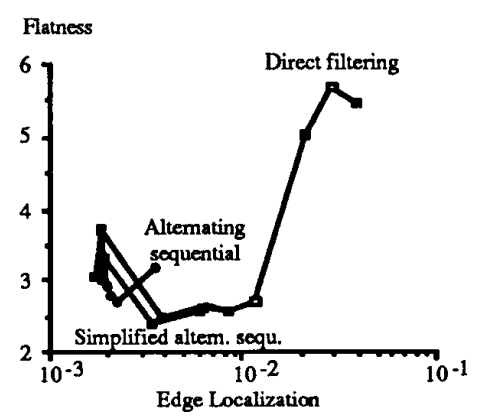

Figure 4: Edge versus Flatness performances of simplified alternating sequential filters 


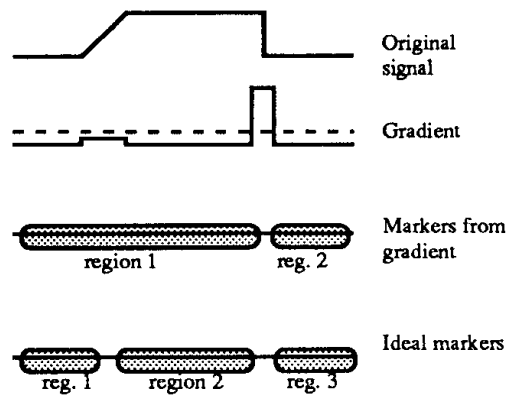

Figure 5: Marker extraction for flat regions

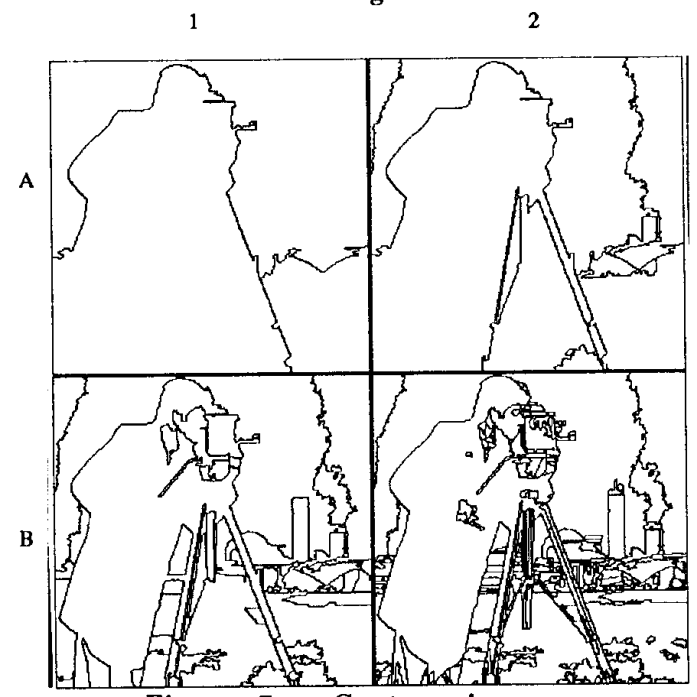

Figure 7.a: Contour image

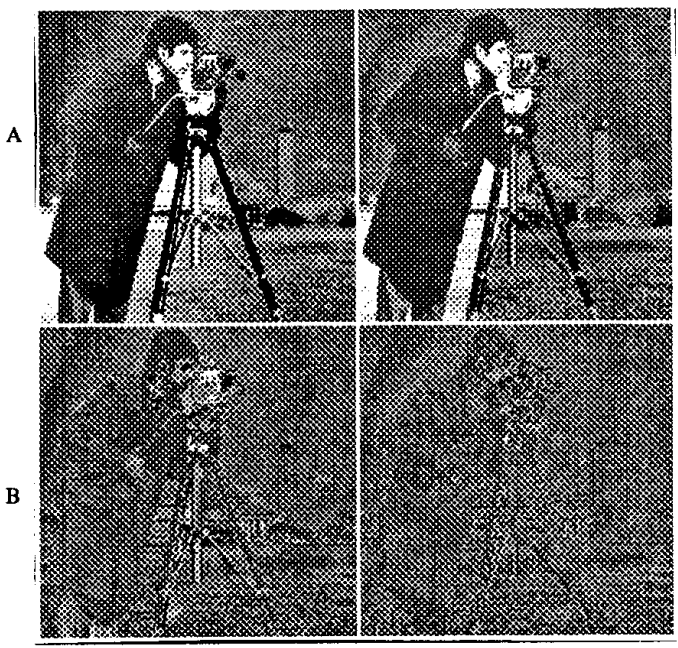

Figure 7.c: Residue images

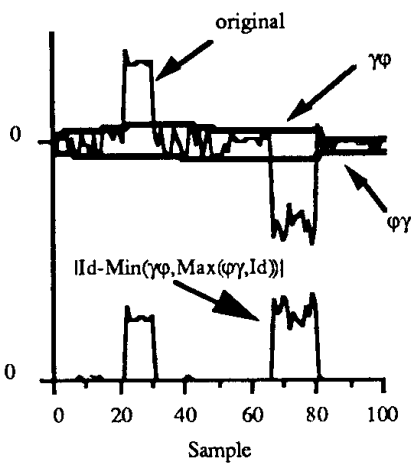

Figure 6: Marker extraction for contrasted regions

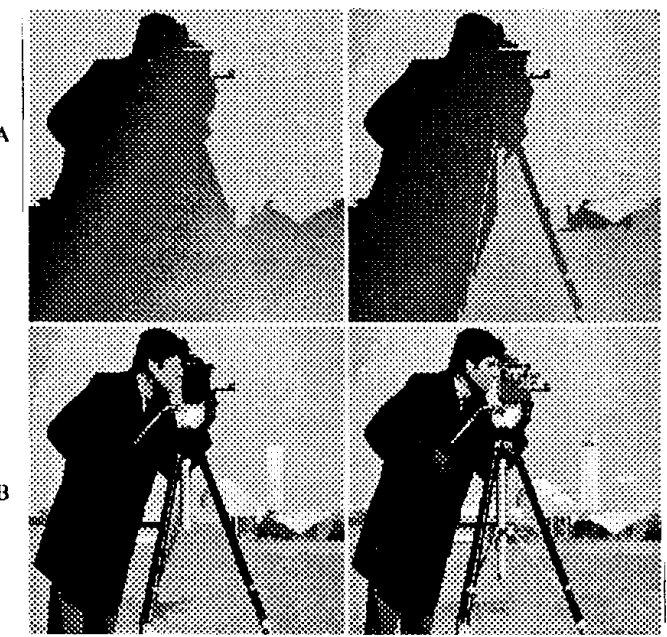

Figure 7.b: Modeled images

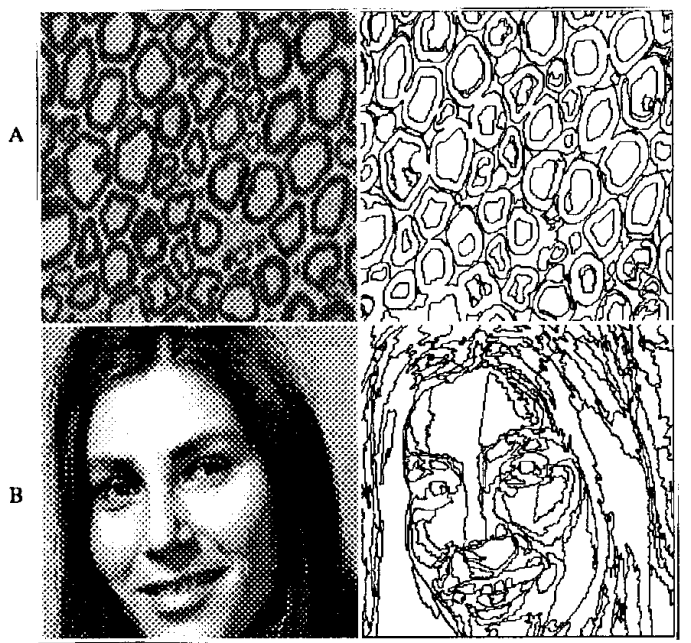

Figure 8: Examples of segmentation 